\title{
Two Kinds of Cognitive Expertise
}

\author{
ELIJAH CHUDNOFF \\ University of Miami
}

\begin{abstract}
Expertise is traditionally classified into perceptual, cognitive, and motor forms. I argue that the empirical research literature on expertise gives us compelling reasons to reject this traditional classification and accept an alternative. According to the alternative I support there is expertise in forming impressions, which further divides into expertise in forming sensory and intellectual impressions, and there is expertise in performing actions, which further divides into expertise in performing mental and bodily actions. The traditional category of cognitive expertise splits into twoexpertise in forming intellectual impressions and expertise in performing mental actions. I consider and address a challenge to my case in favor of adopting this alternative classification of expertise that derives from dual process theories of cognition.
\end{abstract}

Key words: expertise; perceptual expertise; cognitive expertise; motor expertise; mental action

How should we classify the basic forms of expertise? At the most general level there is the superordinate category of expertise. There is some controversy over how best to define expertise. It belongs to a cluster of inter-definable notions: expertise is the capacity for expert performance; expert performance is performance in the manner of an expert rather than a novice; and experts are distinguished from novices by their possession of expertise. Further illumination depends on getting an independent handle on one of the three notions-expertise, expert performance, or expert. Here I will understand expertise in a domain as the capacity for appropriately superior performance in that domain. What counts as appropriately superior performance will depend on the domain and shouldn't be legislated by a general definition of expertise (cf. Ericsson et al 2006, pg. 3, 2018, pg. 4; Gobet 2015, pg. 5)

Individual studies of expertise often focus on specific forms of expertise tied to a domain-e.g. radiology-or to representative tasks-e.g. identifying a lesion. By classifying basic forms of expertise I mean classifying forms of expertise at a level, or select levels, subordinate to the general category-expertise-and superordinate to the various specific domains of expertise or the tasks representative of them. It is difficult to be more precise than this. But it is also unnecessary because there is already a recognized, traditional system of classification at the level that I am interested in and my aim can be adequately understood as that of introducing a competitor to it.

(c) 2019 Wiley Periodicals, Inc. 
The traditional classification is a three-fold classification into perceptual, cognitive, and motor expertise. Merim Bilalić organizes his recent introductory book, The Neuroscience of Expertise, around this traditional classification, and toward the beginning of that book he introduces the classification as follows:

Expertise in radiology requires the visual intake of the information needed for the actual task of spotting lesions within radiological images. As such, it will be used as a typical task of perceptual expertise, relating to domains that predominantly rely on information from our senses. It is evident that experienced radiologists also need to engage their memory, as without it they would hardly be able to spot and categorize lesions. The task itself, however, is a purely visual search task that does not require the mental permutations we find in chess. Chess players rely on the visual information from the chessboard, but for their outstanding performance, they need to go beyond the available visual information. They have to retrieve previously stored chess constellations that may help them to understand the problem at hand, and then, in one of the main aspects of their expertise, to imagine how the game could proceed. Chess is an example of cognitive expertise, where information from our senses plays a secondary role compared to the subsequent engagement of memory and mental simulation. No chess game has been won by just perceiving the situation on the board. Both radiology and chess eventually require motoric responses, either indicating the lesion within a radiological image, or executing a chess move on the board. The motor component in these activities, however, is of no real significance. The essence of sports such as tennis, on the other hand, is exactly the motor component in the performance. Tennis will therefore serve as a prime example of motor expertise, relating to domains that are predominantly shaped by motoric responses. (Bilalić 2017, pgs. 4 - 5; bold print in original) ${ }^{1}$

Bilalic's commitment to the three-fold classification is not without some degree of hesitancy. As he notes, radiologists rely on their medical knowledge about lesions and chess masters rely on their ability to perceptually encode the layout of the chessboard. This hesitancy is widespread. ${ }^{2}$ I believe it is indicative of serious shortcomings in the three-fold classification, some of which I discuss below.

In this paper I will argue that the traditional category of cognitive expertise is disunified. There are two kinds of cognitive expertise: instances of one kind are importantly similar to paradigm cases of perceptual expertise, instances of the other kind are importantly similar to paradigm cases of motor expertise. My main goal in this paper is to substantiate these claims, both by giving reasons for believing them and by elaborating on them with supporting theory. I will propose a system for classifying forms of expertise according to which there is expertise in forming impressions, which further divides into expertise in forming sensory and intellectual impressions, and there is expertise in performing actions, which further divides into expertise in performing mental and bodily actions. The category of cognitive expertise splits into expertise in forming intellectual impressions and expertise in performing mental actions. There is a picture below. After a brief preliminary discussion of the point of arguing over systems of classification in section $(\S 1)$ I introduce my preferred four-fold system for classifying forms of expertise and 
highlight the advantages that I think it has over the three-fold classification. In sections (\$2) and (§3) I support the claims I make on behalf of the four-fold system of classification. Both sections are grounded in the empirical research literature on the psychology of expertise. Section (\$2) calibrates the three-fold and the four-fold systems of classification by focusing on forms of expertise on which the two need not conflict. I make a proposal about the structural differences between expertise in forming impressions and expertise in performing actions. Section (\$3) introduces those tensions that I think favor adopting the four-fold system of classification over the three-fold system of classification. In section (\$4) I bring the foregoing material together into a discussion of how to classify six example forms of expertise. In section (§5) I consider an alternative four-fold system of classification suggested by dual-process theories of cognition.

\section{Systems of Classification}

The project I am engaged in here is similar to but distinct from the project of defending and explaining the distinction between perception and cognition, which has increasingly drawn the attention of philosophers and psychologists (see, for example, Beck 2017, Firestone and Scholl 2016, Orlandi 2014, and Mandelbaum 2017; the increased attention is driven in part by challenges to the distinction, such as those issued in Hohwy 2012, Clark 2013, and Lupyan 2015; Phillips 2017 argues for pluralism about the distinction).

The difference between the two projects is that even though on the traditional classification of expertise there is a division between perceptual expertise and cognitive expertise it is not plausible to identify the fate of this division with that of the distinction between perception and cognition. One can be firmly committed to the distinction between perception and cognition but reject the traditional division between perceptual expertise and cognitive expertise. Suppose psychological states and processes neatly divided into perceptual and cognitive states and processes. Still, it might be that traditionally classified perceptual forms of expertise such as expertise in radiology consist in the presence of both perceptual and cognitive states and the operation of both perceptual and cognitive processes. The same goes for traditionally classified cognitive forms of expertise such as expertise in chess: they might consist in the presence of both perceptual and cognitive states and the operation of both perceptual and cognitive processes. The point continues to hold when we take into account traditionally classified motor forms of expertise such as expertise in tennis. One need not reject the distinctions between perceptual, cognitive, and motor psychological states and processes in order to think that traditionally classified motor forms of expertise consist in the presence and operation of all three sorts of states and processes. In fact the possibilities just described are actual, and that is the major source of the hesitancy with respect to the traditional, three-fold classification, which can be found in the passages quoted above.

The similarity between my project and the project of defending and explaining the distinction between perception and cognition is that both projects are 
rationalized by there being some point to arguing over systems of classification. Some systems of classification are better than others. Systems of classification are better when they highlight real similarities and differences, facilitate good deductive and inductive inferences, and subserve formulating useful and elegant generalizations such as laws, explanations, and norms. Commitment to these claims is sufficient to rationalize arguing over systems of classification and is independent of commitment to controversial metaphysical theses about joints in nature or natural kinds. One can evaluate systems for classifying artifacts such as vehicles as better or worse and therefore engage in rational argumentation about which such system to adopt. My interests in classifying forms of expertise are philosophical, and largely epistemological and methodological. I am interested in the impact that certain forms of expertise have on good reasoning at the level of both individuals and groups-that is to say their rational role and their methodological significance. Though my concerns are primarily epistemological and methodological I intend to characterize a system for classifying forms of expertise that should have broad appeal. It will be motivated by reflection on the empirical psychology of expertise. And its categories will neatly map onto ordinary ways of talking about expertise.

Here, in diagram form, is what I propose:

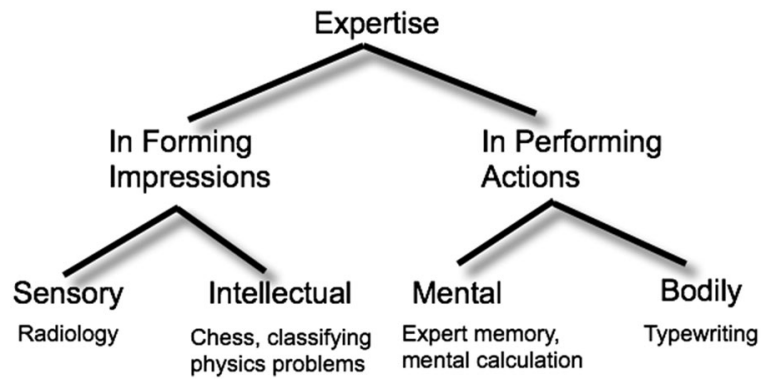

Some clarification of what I have in mind by notions such as impression, action, sensory, intellectual, mental, and bodily is in order.

As I will understand them, impressions are appearances, or seemings. You form an impression that something is a certain way when that thing appears, or seems, to you to be that way. Here I am using "appears" and "seems" in their phenomenal senses rather than their epistemic senses (cf. Brogaard 2013). If you look at a straight stick in water, then in one sense it looks (appears, seems) bent to you, but in another sense it does not. It is the phenomenal sense in which the stick looks (appears, seems) bent to you, and it is the epistemic sense in which it does not. The phenomenal sense tracks the character of your experience; the epistemic sense tracks the direction of your judgment. When, in the phenomenal sense, the stick looks (appears, seems) bent to you, then I understand you to have an impression as of a bent stick, or, less idiomatically, to have formed an impression as of a bent stick. 
Examples of expert sensory impressions include:

Birdwatching: Expert birdwatchers recognize whether a seen animal is a robin faster or as fast as they recognize whether it is a bird. Novices, on the other hand, take more time to recognize whether a seen animal is a robin than they do to recognize whether it is a bird (Tanaka and Taylor 1991).

Radiology: Radiologists can reliably tell whether a seen x-ray image is abnormal without scanning its details. Medical students in training, on the other hand, have to scan the details before reliably making such a judgment (Kundel and Nodine 1975).

And examples of expert intellectual impressions include:

Physics: Experts at solving physics problems initially classify presented problems according the underlying principles governing their solution, such as conservation of energy. Novices, on the other hand, initially classify presented problems according to superficial characteristics, such as involving an inclined plane (Chi et al 1981).

Nursing: Expert nurses can immediately diagnose patients without deliberately taking into account variables such as temperature, blood pressure, pulse, etc. Novices, on the other hand, work out their diagnoses on the basis of guidelines connecting such variables to possible medical conditions (Benner 1982).

It is customary to take birdwatchers and radiologists to illustrate one kind of expertise, expert perception. And it is customary to take experts at solving physics problems and nursing to illustrate another kind of expertise, expert intuition. To a first approximation, the idea is that robins and abnormal x-ray images can be identified by patterns presented to the senses, in these cases vision, but conservation of energy problems and medical conditions cannot be identified without taking into account more abstract patterns grasped by thought.

The two identifications-of expert perception with expertise in forming sensory impressions and of expert intuition with expertise in forming intellectual impressions-together with the gloss on impressions as phenomenal appearances, or seemings, jointly imply two substantive claims. First, expert perceptions are a kind of sensory perceptual experience. Second, expert intuitions are an analogous kind of experience, though not sensory. That these are substantive claims can be seen by considering alternative views on which expert perceptions and expert intuitions are both kinds of judgment, or epistemic seeming, rather than kinds of experience, or phenomenal seeming. The two claims are embroiled in a number of controversies about perceptual experience and intuition that I cannot pursue further here, though I do elsewhere. ${ }^{3}$ I believe the current discussion can be temporarily isolated from those controversies. I will mostly talk about expert sensory and intellectual impressions, not expert perception and expert intuition, in order to facilitate such isolation. The reason I mention the controversies is to highlight that the present discussion is ultimately connected to them, though space considerations prevent further discussion of the connections in this paper. 
Forming an impression is an activity in a broad sense that includes hiccuping and blinking. There is a difference between a stick's looking bent to you and you looking at a stick in order to tell whether it is bent. In the first case you form an impression. In the second case you perform an action, which action might very well result in your forming an impression. In performing an action you pursue a goal. When you look at a stick it is in order to achieve something, e.g. tell whether it is bent or take in the stick's appearance or perhaps just keep your mind off other things. Happening to see the stick and thereby forming some impression of it does not imply looking at the stick. Say you are staring blankly ahead and someone who does happen to be in your visual field asks, "Are you looking at me?" you would be perfectly within your rights to answer, "No," even if you did in fact see the person. In bodily actions a goal is pursued by making your body be a certain way, say by moving your eyes, head, limbs, fingers, or perhaps by keeping still. Signing your name is an example of a bodily action. In mental actions a goal is pursued by making your mind be a certain way, say by rehearsing a list, manipulating a mental image, attending to a consideration, or perhaps by deliberately keeping your mind empty. Silently counting down from ten is an example of a mental action.

In distinguishing expert impressions from expert actions I am not denying that there are important causal links between impressions and actions. Impressions guide actions and actions enable impressions. For example, you need to have a sense of where a tennis serve is heading in order to plan to return it and you need to look at the relevant locations in your visual field in order to gather the cues that will inform the said impression. These interactions between impressions and actions do not show that there are not significant differences between expertise in forming impressions and expertise in performing actions. And they do not show that the proposed classification is just as misleading as the classification of expertise into perceptual, cognitive, and motor forms of expertise.

Classifying expertise in returning a tennis serve, say, as a form of motor expertise rather than a form of perceptual or cognitive expertise is misleading because it suggests that superiority in returning a tennis serve is a function of superiority on some motor skill such as moving one's arms. But this is not the case. Superiority in returning a tennis serve is a function of the superior orchestration of an assortment of perceptual, cognitive, and motor skills such as engaging in visual search, using working memory, and timing movements in one's limbs (cf. McPherson and Thomas 1989; McPherson and French 1991; McPherson 2000; for recent philosophical discussion of the general point: Stanley and Krakauer 2013; Toner et al 2015; Christensen et al 2016; Montero 2016; Fridland 2014, 2017). Classifying expertise in returning a tennis serve as a form of expert bodily action is not similarly misleading because there is no temptation to think of acting as simply a matter of motor skill. In general when we act we depend on an assortment of perceptual, cognitive, and motor skills. And there is no reason to think that expert action, including specifically expert bodily action, is any different.

One of the main advantages of classifying forms of expertise in the way I am proposing is that it highlights similarities and differences that the more traditional three-fold classification obscures. The major shifts in conceptualization occur with 
respect to forms of expertise traditionally classified as cognitive. Consider two examples: expertise in classifying physics problems and expertise in doing multidigit multiplication in one's head. The three-fold classification joins these under the category of cognitive expertise and separates them from forms of perceptual expertise such as expertise in detecting lesions in an x-ray image and forms of motor expertise such as expertise in typewriting. Here are the similarities and differences that I believe this obscures:

(A) There is a similarity between classifying physics problems and detecting a lesion in an x-ray image. Both are impressions of patterns. There is also a distinguishing difference: one is an intellectual impression and the other is a sensory impression. But it is important, I think, to note the root similarity, and the three-fold classification does not.

(B) There is a similarity between doing multi-digit multiplication in one's head and typewriting. Both are complex actions requiring selecting and executing the right procedures. There is also a distinguishing difference: one is a mental action where the procedures are algorithms for manipulating one's mental representations and the other is a bodily action where the procedures are motor plans for moving one's fingers. But it is important, I think, to note the root similarity, and the three-fold classification does not.

(C) Noting the similarity between classifying physics problems and detecting lesions in an x-ray image, on the one hand, and the similarity between doing multi-digit multiplication in one's head and typewriting, on the other, highlights a crucial difference between classifying physics problems and doing multi-digit multiplication in one's head. One is forming an impression and the other is performing an action. Highlighting this difference is, I think, more important than recognizing that both draw on cognitive skills, since detecting a lesion in an x-ray image and typewriting also draw on cognitive skills. ${ }^{4}$

I do not intend the above remarks to stand alone. In the next two sections I review some of the empirical research literature on the psychology of expertise. I believe that reflecting on this research will bring out some support for the contentions that I have put forward in (A) through (C).

\section{Expert Impressions and Expert Actions}

In this section I will propose structural differences between expertise in forming impressions and expertise in performing actions. I will proceed by extrapolating the structural differences from reflection on two paradigm cases. Expertise in radiology is my paradigm case of expertise in forming impressions and expertise in typewriting is my paradigm case of expertise in performing actions.

\section{Radiology}

Studies contrasting expert radiologists with novices such as medical students have demonstrated that expert radiologists are more efficient at examining x-ray images than are novices in a number of measurable ways. One simple measurement is that 
experts spend less time examining x-ray images. They find more abnormalities or correctly judge $\mathrm{x}$-ray images to contain no abnormalities in a shorter amount of time. A good deal of evidence about the processes underlying this efficiency comes from eye-tracking studies that measure efficiencies in the scanpaths of expert radiologists. As experts scan x-ray images they fixate on fewer locations and so make fewer saccades, i.e. movements of eyes between fixation points. The saccade lengths are longer, but the overall scanpath length is shorter. That is, the distance between one fixation and the next is longer but the overall distance covered by the series of fixations is shorter. The time it takes experts to first fixate on abnormalities in x-ray images is shorter. The proportion of time spent fixating on abnormalities increases with expertise. Overall expert's dwell on x-ray image locations for shorter periods of time, but spend relatively longer periods of time dwelling on locations containing abnormalities. Finally, experts fixate for shorter periods of time and the rate of fixation increases with expertise. (See Sheridan and Reingold 2017, pg 5 for a tabulation of these results along with extensive references to the literature demonstrating them.)

Instead of listing additional empirical phenomena associated with expertise in radiology I believe it will be more helpful at this point to consider a psychological model of what underlies this expertise. According to the global-focal search model, expert radiologists form impressions about the presence and location of abnormalities in an x-ray image by rapidly making a global comparison between the seen x-ray image and a stored schema of a normal x-ray image, which comparison then informs a focal search of those areas where the seen image deviates from the stored schema. Nodine and Kundel, the primary architects of this model, diagram it as follows:

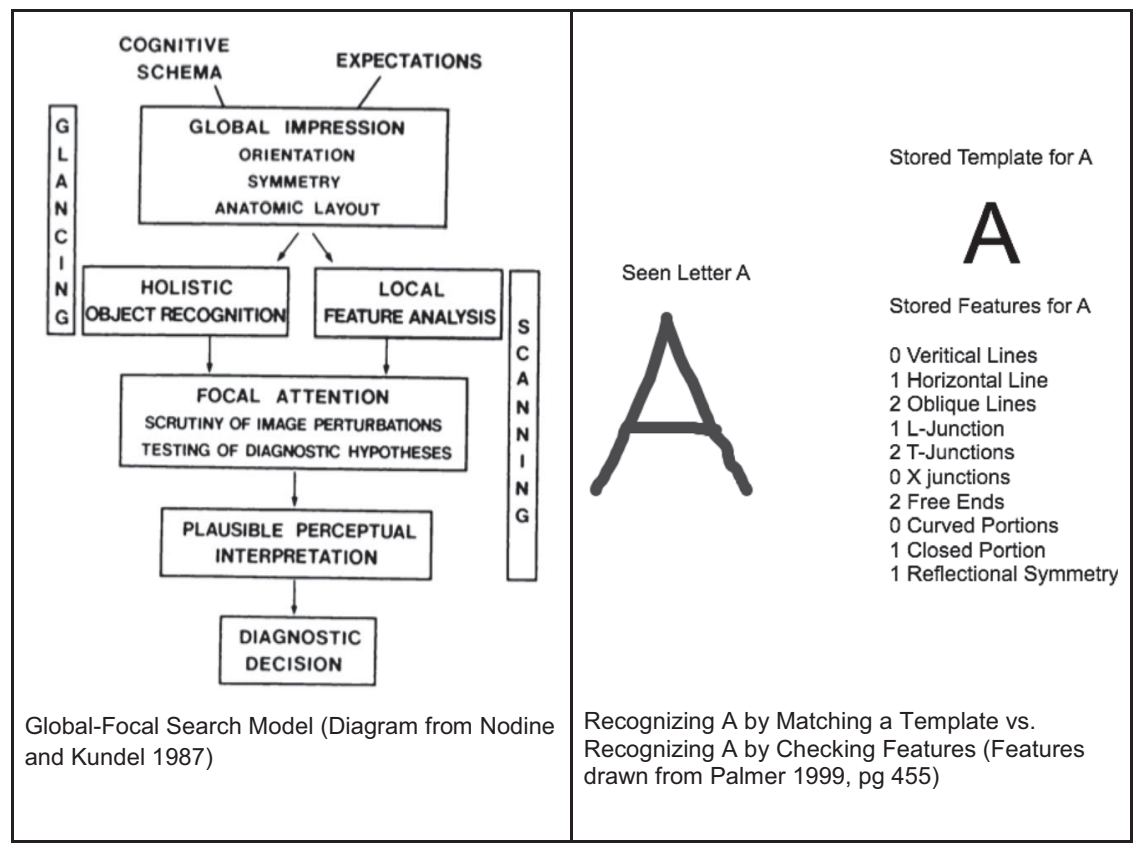


The global-focal search model is one among a number of models of expertise in medical image perception that accord holistic perceptual processing a prominent role in explaining expert performance (see Sheridan and Reingold 2017 for a review of others). This is in line with a growing consensus, which first began to emerge in the study of face perception, that superior perceptual recognition skills in general derive from an increased reliance on holistic perceptual processing (see Gauthier et al 2010 for a book length review).

I will not attempt a full exploration of holistic perceptual processing here. To get a sense for what it is consider two ways of recognizing whether a seen letter is the letter A. On the template matching approach you compare the seen letter with a stored template for the letter A. The comparison is holistic in that you do not compare the two bit by bit, but as wholes. On the feature analysis approach you check the seen letter for the presence of those features that characterize the letter $\mathrm{A}$ (see above). The checking proceeds bit by bit. Template matching is just one kind of holistic processing, and no simple form of it is psychologically realistic, but it is a useful paradigm to keep in mind. It can usefully inform our understanding of the global-focal search model of radiological expertise. Imagine you are examining images of the letter A for abnormalities. It would be more efficient to try to match a seen letter $\mathrm{A}$ with a template for a normal letter $\mathrm{A}$ and then check the areas of mismatch for abnormal features, say one insufficiently oblique line that fails to align with the template, than it would be to begin by just checking the features one by one. The essence of the global-focal search model, and other holistic models, is that this is what expert radiologists are able to do. Expert radiologists are superior in forming impressions of $\mathrm{x}$-ray images because they rapidly figure out where to look (by something like template matching) and they know what to look for once looking there (by something like feature checking).

Consider how the global-focal search model explains some of the empirical observations about expert radiological performance reviewed above. Radiologists find more abnormalities or correctly judge x-ray images to contain no abnormalities in a shorter amount of time. Why? Because the global schema comparison stage returns an early verdict of normality or highlights the abnormal locations. As experts scan x-ray images they fixate on fewer locations. Why? Because they just bother with the locations highlighted by the global schema comparison. Experts' saccade lengths are longer, but the overall scanpath length is shorter. Why? Because in their guided search they can skip around to just those locations highlighted by the global schema comparison and perhaps in a way informed by a learned priority ranking. Overall experts dwell on x-ray image locations for shorter periods of time, but spend relatively longer periods of time dwelling on locations containing abnormalities. Why? Because they have stored property representations they can use in local feature analysis, and they only use them for those locations marked as abnormal.

\section{Typewriting}

Experts in transcription typewriting type faster than novices in transcription typewriting. As Gentner notes in an influential study of this form of expertise, the 
question is: "Is expert performance simply a speeded up version of student performance, or do qualitative changes in performance occur during the acquisition of typing skill?" (Gentner 1988, pg. 5). Useful information bearing on this question comes from studying the differential effects of expertise on typing four kinds of digraphs: repeated letters such as "dd" (doubles), non-doubles typed using one finger such as "de" (1-finger digraphs), two letters typed by two fingers on the same hand such as "dr" (2-finger digraphs), and two letters typed by fingers on different hands such as "do" (2-hand digraphs). What Gentner found is that while experts type all digraphs faster than novices, the increase in speed is significantly different depending on the kind of digraph in question. The speed is measured by interstroke intervals: the shorter the interstroke interval, the faster the digraph is typed. The interstroke intervals for typing doubles showed the least dramatic change with expertise; the interstroke intervals for typing 2-hand digraphs showed the most dramatic change; and the interstroke intervals for typing 1-finger and 2-finger digraphs showed intermediate changes, with 2-finger digraphs showing a greater decrease in interstroke intervals than 1-finger digraphs.

Two related phenomena are worth mentioning. First, in general the time it takes to type the next letter in transcription typing is much faster than the time it takes to type the same letter as a response to some stimulus. That is, people-both novices and experts-are much faster at typing "o" after "w" while typing "word" than they are at typing "o" in response to some stimulus, say a chime. Second, in general performance on transcription typing slows down considerably when people-both novices and experts-are prevented from being able to look ahead in the text to be transcribed. (Both phenomena, along with many others, are reported in Salthouse 1986). These two phenomena support overlapping processing models of transcription typing. According to these models the processes responsible for typing successive letters overlap. So as you are pressing the "w" key concurrent psychological processes are unfolding that will result in you pressing "o" then " $\mathrm{r}$ " then "d," say. Salthouse distinguishes four components of transcription typewriting and provides the following diagram:

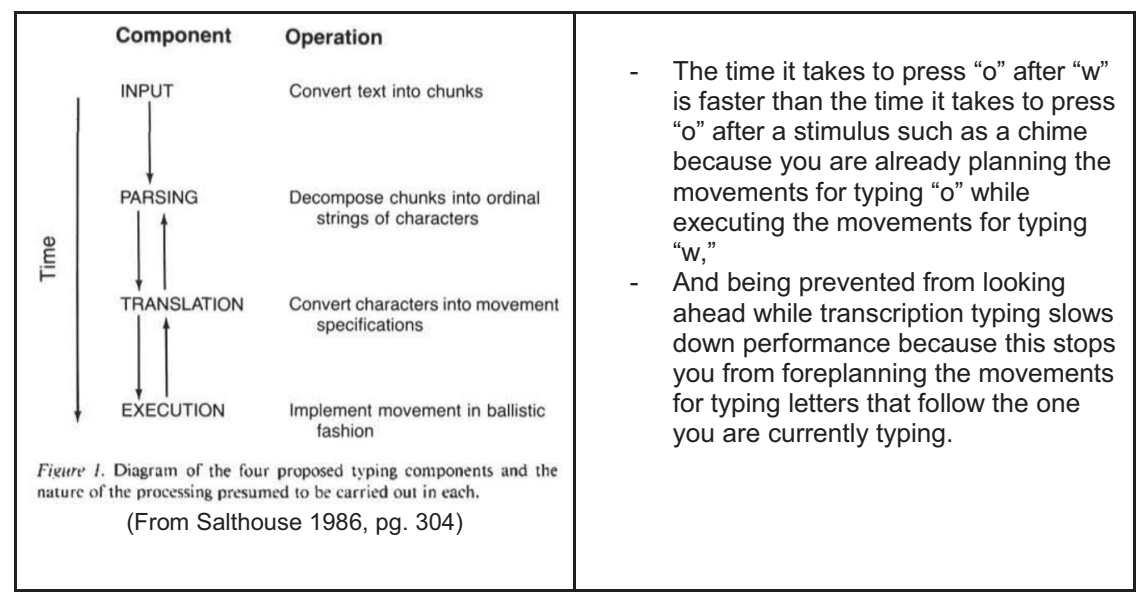


Overlapping processing models of transcription typing provide a nice setting within which to understand the unequal distribution of performance improvements that experts show in typing different kinds of digraphs. The distribution comes from the degrees of overlap that the different kinds of digraphs allow. 2-hand digraphs allow the greatest overlap because you can start moving the finger to type the second character while pressing the key for the first character. 2-finger digraphs also allow this to some extent. Further, experts learn to move the finger typing the first character in 2-finger digraphs in such a way as to set up rather than disrupt the movement of the finger typing the second character. 1-finger digraphs and doubles do not allow overlapping movements, but they do allow for overlap between moving the finger typing the first character and planning to move the finger typing the second character. (See Gentner 1988 for multiple sources of evidence documenting that experts capitalize on these opportunities for overlapping processing). These findings about expertise in typewriting fit with similar findings about expertise in other activities such as those in various sports, where anticipation and strategic planning are also observed to play a large role in accounting for experts' superior performance. (See Yarrow 2009 for a review).

\section{Structural Differences}

I propose that expertise in forming impressions and expertise in performing actions are structurally different kinds of expertise. A capacity for searching is central to the first; a capacity for planning is central to the second. Further elaboration on these claims follows.

Expertise in forming impressions about a domain is a capacity for forming superior impressions about that domain that is grounded in domain related search strategies. Two clarifications are in order. First, I am working with a very general notion of search. Search is possible whenever it is possible to gather information by exploring paths in a space. The space and the paths might be concrete: the space might consist of image locations and the path connections might consist of saccades. Or the space and the paths might be abstract: the space might consist of possible arrangements of a chess board and the path connections might consist of legal chess moves. Second, I do not mean to say that every expert impression is the result of searching activity. Rather, I am suggesting that every expert impression is the result of a capacity that is grounded in the domain related search strategies that are in one's possession. That capacity can be superior along a preparatory, pre-search dimension.

Whenever you search you (1) represent a space to be searched, (2) explore paths in that space, and (3) gather information from the paths explored. Suppose you are looking for your keys in your bedroom. You represent your bedroom as a set of locations. You explore paths consisting of sets of those locations. And you gather information from those paths, namely information about which do and which do not contain the location of your keys. Search strategies can result in superior impressions because of improvements along any one of these dimensions. Consider, again, the global-focal search model of radiological expertise. The global schema comparison results in a representation of the space to be searched partly as a set 
of deviations from a normal x-ray image. Radiologists prioritize the paths through this space that are more likely to contain significant information. And radiologists develop feature analyzers that extract this information from the paths explored. The impressions that radiologists form of $\mathrm{x}$-ray images are grounded in their possession of this distinctive strategy for searching x-ray images. But a radiologist might form an impression of an x-ray image, for example an impression as of its abnormality, without actually engaging in any search (cf. Kundel and Nodine 1975). If the radiologist represents the $\mathrm{x}$-ray image partly as a set of deviations from a normal $\mathrm{x}$-ray image, then he or she can form an impression as of its abornality just by virtue of this set being non-empty and without having to search the $\mathrm{x}$-ray image for specific abnormalities. ${ }^{5}$

Expertise in performing actions in a domain, on the other hand, is a capacity for performing superior actions in that domain that is grounded in domain related planning strategies. Planning might be dynamically intertwined with the action planned. That is, the plan for an action need not be complete before the action is initiated and it might be updated in light of feedback gained while performing the action. It is useful to think of execution as a phase of planned action rather than something separate. So when you plan an action you (1) represent a goal to be achieved, (2) compose a list of tasks whose execution will achieve that goal, and (3) execute the tasks on that list. Planned action can be superior because of improvements along any one of these dimensions. Consider, again, expert transcription typewriting. Experts anticipate upcoming typing goals by looking ahead and experts plan the movements of their fingers so that the movements at one time facilitate and do not disrupt the movements that will be required at a later time. Further, experts execute the planned movements faster and with fewer errors, such as switching around pairs of letters or pressing a key next to the intended one. Typewriting provides a particularly simple environment in which to study expert action. The task itself is simple compared to the more spectacular performances of athletes. And the performance of it is easier to measure since it is relatively easy to time key presses and to verify the accuracy of a transcription. Nonetheless I think expert transcription typewriting is representative with respect to some key structural aspects of expertise in performing actions that distinguish this form of expertise from expertise in forming impressions.

\section{Two Kinds of Cognitive Expertise}

Superior strategy plays a role in explaining both the expert impressions that radiologists form and the expert actions that transcription typists perform. But the strategy takes different forms. Expert radiologists are superior at strategically searching $\mathrm{x}$-ray images. They capitalize on the information available in an initial glance and use that information to guide their more psychologically costly search of the image's details. Transcription typists are superior at strategically planning their finger movements. They arrange their current finger movements to reduce the effort required for later finger movements and they do as much advanced psychological processing 
of those later finger movements as is compatible with physical constraints and their level of expertise.

Reflection on both radiology and transcription typewriting suggests a natural division in forms of expertise, with expertise in radiology lying on one side and expertise in transcription typing lying on the other. But this is not surprising, and it does not yet speak in favor of my proposed four-fold classification of forms of expertise. The traditional three-fold classification already draws a division between the two, classifying expertise in radiology as perceptual and classifying expertise in transcription typewriting as motor. The case for the new four-fold classification only emerges when we compare these two forms of expertise with at least two others, both traditionally being classified as cognitive but one bearing greater similarity to expertise in radiology and one bearing greater similarity to expertise in transcription typewriting. That is the aim of this section. I will consider a total of four forms of expertise that are traditionally classified as cognitive: chess, physics, memory, and calculation.

\section{Chess and Physics}

Research on chess perception has been a mainstay in research on expertise since the pioneering work of (De Groot 1965) and (Chase and Simon 1973). A series of studies conducted by Charness, Reingold and their collaborators aims to use the same sorts of eye-tracking evidence that is used in exploring expertise in radiology to explore expertise in chess. The results are strikingly similar (see Reingold and Charness 2005 and Reingold and Sheridan 2011 for reviews; the latter explicitly draws parallels between expertise in radiology and expertise in chess). For example, experts in chess have larger visual span for chess-related configurations but not for visual patterns in general, where visual span measures the area from which information is extracted during an eye-fixation. Experts in chess make fewer fixations when engaged in the task of detecting whether a king is in check, and a smaller proportion of their fixations fall on pieces rather than between pieces (which is taken as an indication of holistic processing).

(Reingold et al 2001) collected this data both for check detection tasks performed on boards that represented pieces using the more familiar pictorial symbols for chess pieces and on boards that represented pieces using letters for chess pieces, such as "K" for the king. They found that experts' advantage was greater with the the more familiar pictorial symbols, but still existed with the letters. In their review Charness and Reingold write that this demonstrates "that the experts' encoding advantage is related at least in part to their chess expertise, rather than to a general perceptual superiority" (338). To my mind it also shows something else, namely that chess experts are forming intellectual impressions in addition to sensory impressions of the chess board. This is a point worth emphasizing because it wouldn't serve my purposes if these studies are probing a form of expertise that should count, along with expertise in radiology, as expertise in forming sensory impressions. I believe chess expertise does include superior formation of sensory impressions. But the evidence seems to me to suggest that it also includes superior formation of intellectual impressions. Sensory encoding enables these impressions, but they are 
not of patterns in sensory data, but rather of patterns in more abstract data grasped by the intellect. Compare grasping the pattern in the following sequence of numbers, " $2,4,6,8,10, \ldots$ " You need to see the numerals to grasp the pattern, but the pattern is in the numbers not the numerals.

Charness and Reingold distinguish between two phases in encoding chess positions. In the first players encode the identities and locations of pieces. And in the second players take that information and extract further information about chess relations. In a series of experiments they found evidence that chess experts extract this further information both automatically and in parallel. The evidence includes the fact that expert performance in this second phase is not reduced by the addition of extra pieces to process and does not benefit from cueing. Further, expert performance in this second phase exhibits interference effects that have been taken as a hallmark of automaticity ever since Stroop's pioneering work (Stroop 1935). Finally, check detection is one task, but choosing the next move in a game is another, ecologically more valid task. Charness and Reingold review some of their studies on this task as well. They found that the fixations experts make in their initial glance at the board fall on more empty squares (indicating holistic processing), and for those fixations that do fall on pieces, a greater proportion fall on strategically significant pieces. In other studies they showed that when selecting the next move experts make fewer fixations and a greater proportion of longer fixations as the task progresses in time, suggesting, as the similar results for radiologists did, earlier fixations on locations with useful information guided by the overall information available in the initial glance.

I believe that expertise in chess includes expertise in forming intellectual impressions about chess relations such as checking. The similarity between this form of expertise and the expertise that radiologists have in forming sensory impressions about abnormalities in x-ray images should be evident from the research I have reviewed. Much of it is motivated precisely by the idea that there are these similarities. I will try to make them sharper after discussing expertise in physics, memory, and mental calculation.

(Chi et al 1981) asked novice and expert physics problem solvers to group physics problems according to similarity of solution, without giving them time to actually solve the problems. The study elicited impressions of similarity and difference between physics problems. What Chi et al found is that novices and physicists generated different groupings, corresponding to different impressions of similarity and difference. Novices grouped problems according to surface structure-the objects mentioned (inclined plane), their configurations (block on top of inclined plane), and the physics terms used ("friction"). Experts grouped problems according to the physical principles governing their solution, such as conservation of energy or $\mathrm{F}=\mathrm{MA}$. Chi et al provide a model of how expert physics problem solvers form their impressions of physics problem that is similar to Nodine and Kundel's global-focal search model of expertise in radiology. Because of their extensive domain related knowledge, experts' initial review of problem statements cues potential physical principles governing their solutions and associated schemata for applying those principle; these physical principles and schemata guide experts' subsequent 
examination of the problem for information that will fill out a solution strategy (cf. Chi et al 1981, pg 145). It is also worth highlighting a similarity between the (Chi et al 1981) task of sorting physics problems without solving them and the (Kundel and Nodine 1975) task of making fast judgments about x-ray image normality or abnormality. In both cases there is not enough time to engage in searching activity-in the physicists' case searching the problem space for a solution, in the radiologists' case searching the $\mathrm{x}$-ray image for specific abnormalities. Nonetheless, the superior impressions can be grounded in improved domain related search strategies in the sense elaborated in the previous section, since there I allowed that the operative improvement might be along a preparatory, pre-search dimension-e.g. in how locations or problems are represented as a set up for, but still prior to searching activity. See (Chi 2011 and Nokes et al 2011) for reviews of more recent literature on expertise in problem solving that emphasize this aspect of it.

If there was any doubt about whether chess impressions adequately illustrate intellectual as opposed to sensory impressions, no such doubts should apply to the case of physics. It would be difficult to argue that expert physics problem solvers are forming sensory impressions of sensible patterns in physics problems. The research shows that this is exactly what they are not doing: rather they are forming impressions of patterns in physics problems that only emerge at the abstract level of physical principles. Given that we have this independent reason to think there are expert intellectual impressions in physics, then I see no bar to thinking that chess experts also form such impressions of abstract chess relations, in addition to impressions of the more concrete sensible patterns in chess pieces that realize those relations.

\section{Memory and Calculation}

Now let's consider two mental actions: keeping a sequence of numbers in mind, and multiplying multi-digit numbers without use of external memory aids. One is more basic than the other since you have to keep a sequence of numbers in mind in order to keep track of your work as you multiply multi-digit numbers. The usual, novice method of keeping a sequence of numbers in mind is rehearsing it to oneself. This method works if the sequence is no longer than a handful of numbers but fails thereafter. The reason is twofold. First, though working memory encoding and retrieval times are fast, its storage capacity is severely limited. ${ }^{6}$ Long term memory storage capacity is-for all we know, and for all practical purposesunlimited, but encoding and retrieval times are slow. If working memory were unlimited, then rehearsal would allow one to keep more numbers in it for ready access. If long term memory encoding and retrieval were faster, then the numbers to be kept in mind could be placed directly in it and readily accessed as needed.

Expert mnemonists keep much longer sequences of numbers in mind by strategically overcoming the typical constraints on working memory and long term memory. Suppose I read out the following sequence of numbers and ask you to keep them in mind and repeat the sequence after I finish: $1,8,6,1,1,9,1,4,1,9,3$, 
$9,1,8,6,5,1,9,1,8,1,9,4,5$. Unless you catch the trick, likely you'd find the task impossible. But suppose I read out the same sequence after telling you that I will read out three years in which a war started-Civil, WWI, and WWII-and three years in which a war ended-again Civl, WWI, and WWII. Then likely you'd find the task easy. The first task is impossible because working memory is too limited in capacity and long term memory encoding and retrieval are too slow. The second task is easy because working memory is capacious enough to keep in mind that you are to recall the starting and then the ending years of the Civil War, WWI, and WWII, and you already have those starting and ending years stored and indexed to these names in long term memory from previous learning. Theories of expert memory differ in their details but the contrast between these two tasks and these observations about the source of that contrast brings into focus their common core. Expert mnemonists in a domain-and expert memory is always relative to a domain such as numbers-store domain related structures in long term memory and strategically organize information in working memory so that it connects to those structures. (Chase and Ericsson 1982) contains pioneering early studies on expert memory and a theoretical framework for understanding them; (Ericsson and Kintsch 1995) and (Gobet and Simon 1996) present more elaborate theories that deal with further experimental results.

We all learn a simple algorithm for doing multi-digit multiplication, but multidigit mental multiplication remains a difficult task for novices because, without external memory aids, the algorithm requires us to keep operands and intermediate results in mind while proceeding with computations. Expert mental calculators do better largely by planning out more efficient problem specific strategies that exploit specialized algorithms and a store of arithmetical and algebraic facts (Staszewski 1988; Pesenti 1999). Knowing the squares of double digit numbers and the difference of squares formula- $\mathrm{a}^{2}-\mathrm{b}^{2}=(\mathrm{a}+\mathrm{b})(\mathrm{a}-\mathrm{b})$-for example, would allow a fast solution of $24 \times 36$ since $24 \times 36=(30-6) \times(30+6)=30^{2}-6^{2}=900-36$ $=864$ (cf. Ericsson and Charness 1994).

Expertise in recalling sequences of numbers and expertise in mental multiplication bear comparison with expertise in typewriting. All three are forms of expert action, and as such all three depend on strategic planning in the use of one's faculties. Expert typewriting depends on strategic planning in finger movements. Expert memory depends on strategic planning in how one is going to organize information so as to be able to store all of it and retrieve the right portions of it as needed. Expert mental calculation depends on strategic planning in how one is going to approach a problem. In these second two forms of expertise the strategic planning is not directed at how one will manipulate one's body parts, but rather in how one will manipulate one's mental representations. Hence they are expertise in performing mental actions rather than in performing bodily action. But the root commonality is that they are expertise in performing actions and this sets them apart from expertise in chess and expertise in classifying physics problems, though all four are traditionally lumped together as forms of cognitive expertise. 


\section{Classifying Six Forms of Expertise}

I've discussed six examples of expertise. Here is how the traditional three-fold classification would arrange them:

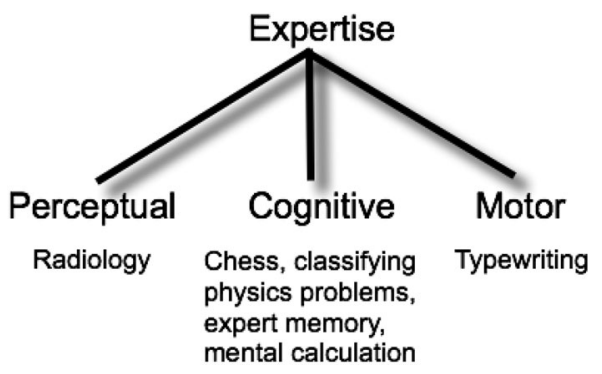

And here is how my proposed four-fold classification would arrange them:

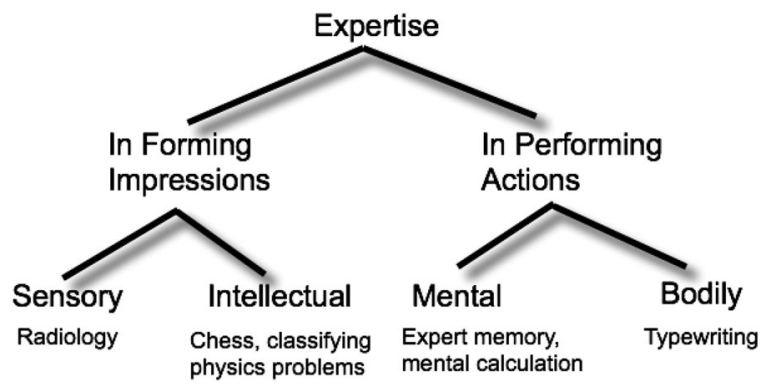

I believe that the research into the different forms of expertise I have reviewed favors my proposed classification. Here I return to the claims about similarity and difference encoded in the diagram and made under (A) through (C) in section 1.

Above I made two claims about the structure of expertise in forming impressions. First, expertise in forming impressions about a domain is a capacity for forming superior impressions about that domain that is grounded in domain related search strategies. Second, whenever you search you (1) represent a space to be searched, (2) explore paths in that space, and (3) gather information from the paths explored. The root similarity between expertise in radiology, chess perception, and classifying physics problems is that all are expertise in forming impressions. This was claim (A) from section 1 . The similarity should show itself in common structural characteristics. And I think we do find that. Radiologists holistically process seen x-ray images and thereby form a representation of the space covered by them in a way that facilitates subsequent search. Chess experts also represent spaces. There is the board containing the chess pieces and there is the space of possible moves. The research on chess expertise suggests that, like radiologists, they benefit from superior representations of these spaces. They represent the distribution of pieces on the board holistically, and they represent the space of possible moves in a way that prioritizes optimal moves. Experts at solving physics problems represent problem 
spaces. They represent those spaces as generated from the underlying structure of a given physics problem rather than its superficial characteristics. This representation on its own can explain expert patterns in the classification of physics problems, which classifications are prior to any actual searching of the problem space so represented.

Above I made two claims about the structure of expertise in performing actions. First, expertise in performing actions in a domain, on the other hand, is a capacity for performing superior actions in that domain that is grounded in domain related planning strategies. Second, when you plan an action you (1) represent a goal to be achieved, (2) compose a list of tasks whose execution will achieve that goal, and (3) execute the tasks on that list. The root similarity between expertise in typewriting, memory, and mental calculation is that all are expertise in performing actions. This was claim (B) from section 1. Again, the similarity should show itself in common structural characteristics. And I think we do find that as well. Efficient planning is central to all three forms of expertise. Expert transcription typists form more determinate representations of finger movements that will be required and plan current finger movements so as to facilitate rather than disrupt their satisfaction. Mnemonists also plan ahead so as to minimize the effort they must expend on a given task. As they are given a list of numbers to repeat they join earlier items with later items into meaningful groups that can be kept in mind with the effort required for a single item. Planning ahead here does not involve somehow looking forward in time, but rather temporarily keeping items from an earlier time in mind till they can be unified with the right later items. Experts at mental calculation also plan by organizing their approach to a given calculation so as to minimize the load placed on their working memory.

So far I've returned to claims (A) and (B) from section 1. Claim (C) was: there is a root difference between expertise in chess and classifying physics problems on the one hand and expertise in memory and mental calculation on the other hand. I think this just follows from (A) and (B) and the structural differences between expertise in forming impressions and expertise in performing actions. Here I want to reiterate a point about cognition, however. Even though the proposed four-fold classification does not label expertise in chess, physics, memory, and mental calculation as forms of cognitive expertise, it does not follow that there is something about these examples of expertise that it obscures. The four-fold system of classification is offered after having already noted that all forms of expertise involve cognition, including these four as well as expertise in radiology and typewriting.

\section{Dual-Process Theories of Cognition}

The three-fold classification of expertise is into perceptual, cognitive, and motor. The four-fold classification of expertise that I have proposed is into forming sensory impressions (perceptual), forming intellectual impressions (cognitive of one kind), performing mental actions (cognitive of another kind), and performing bodily actions (motor). The difference between them is not just in the number of forms of expertise. The classification I have proposed has structure to it that represents the 
similarities and differences reviewed in the previous section. So one might agree that there are four forms of expertise but reject the particular classification that I have proposed. In fact, one might worry that the proposal I have been elaborating is otiose because there is an alternative four-fold classification that has already been worked out in the literature. This is a classification that derives from dual-process theories of cognition.

According to the dual-process theory advocated in (Evans and Stanovich 2013) there are two types of cognitive processes: type 1 processes are essentially autonomous in that they are executed in response to triggering conditions independently of central control; type 2 processes essentially make demands on our limited capacity working memory. Type 1 processes also tend to be fast, high capacity, parallel, nonconscious, biased, contextualized, automatic, associative, guided by prior experience, and independent of individual cognitive ability, but unlike autonomy none of these features is essential. Type 2 processes also tend to be slow, limited capacity, serial, conscious, normatively correct, abstract, controlled, rulebased, guided by anticipated consequences, and correlated with individual cognitive ability, but unlike making demands on working memory none of these features is essential. The two types of processes interact in the following way: type 1 cognitive processes generate default cognitive responses, which may or may not be overruled by type 2 cognitive processes. Other dual process theories of cognition vary along a number of dimensions but have the same basic structure, which is all that will matter here (see Evans and Stanovich 2013 for discussion of the differences and references to the literature).

The challenge, then, is this. Suppose we just keep the traditional classification of forms of experience into perceptual, cognitive, and motor, but note that since cognition itself splits into type 1 processes and type 2 processes there will be two forms of cognitive expertise-expertise in type 1 cognitive processing and expertise in type 2 cognitive processing. In diagram form and with the example forms of expertise placed in their likely places we have:

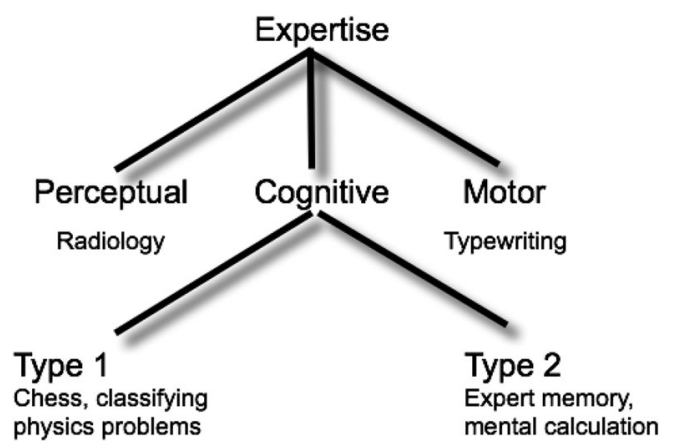

I think there is some prima facie plausibility to this system for classifying forms of expertise, and it has the merits of according both with the tradition of classifying expertise into perceptual, cognitive, and motor, and with a popular, empirically supported body of theorizing about the nature of cognition. That said, there 
are at least four considerations deriving from the present discussion that count against it.

First, it fails to capture the similarities between expertise in radiology on the one hand and expertise in chess and expertise in classifying physics problems on the other. The research literature on these forms of expertise shows that there are real similarities between them. There should be some common root, such as expertise in forming impressions, from which they descend.

Second, it is doubtful that classifying physics problems is a type 1 cognitive process. Chi et al note that experts spent more time than novices did when first sorting a list of physics problems (Chi et al 1981, pg. 124). It wouldn't do to count this form of expertise as a type 2 cognitive expertise, however. Because then the classification would fail to capture the similarity between it and expertise in chess, and it would fail to capture the difference between classifying physics problems on the one hand and expert memory and mental calculation on the other.

Third, while exercising expert memory skills does place demands on working memory there are aspects of expert memory, such as practiced mnemonic strategies, that become automatic and so do not themselves place demands on working memory. A proponent of the system of classification under consideration might argue that these aspects of expert memory should be classified as type 1 cognitive expertise. But then what seemed to be a unified form of expertise turns out to be disunified in a rather unnatural way. Thinking of expert memory as a form of expert mental action does not have this outcome. One action can have automatic and controlled aspects.

Fourth, the system of classification under consideration inherits one of the major problems with the traditional system of classification of expertise into perceptual, cognitive, and motor: it fails to reflect facts such as that expertise in radiology does not just consist in superior perceptual skills but also requires cognitive processing, that expertise in chess does not just result in superior cognitive skills but also manifests in superior perceptual encoding, and that expertise in typewriting is not just a function of superior motor skills but largely depends on perceptual and cognitive processes. If we adopt the system of classification under consideration, then, as (Yarrow et al 2009, pg. 585) point out it is likely to serve only heuristic purposes and not as a guide to research and theory.

These four considerations flow from points made earlier in the paper, and they simply tell against using dual-process theories of cognition to complicate the traditional classification of expertise into perceptual, cognitive, and motor. It has not been my intention here to introduce substantively new material on expertise, nor to challenge dual-process theories of cognition on their own merits. ${ }^{7}$

\section{Notes}

${ }^{1}$ Compare: "Most of the experimental tasks used to study skill focus on the speed and/or accuracy of performance. For example, the speed and accuracy with which problems can be solved under different conditions would be of interest to the investigator of cognitive skill. A motor skills researcher might measure the time to initiate a movement, as well as the time to execute the movement and its accuracy, and a researcher investigating a predominantly perceptual skill would measure accuracy of classification 
or perceptual thresholds." (Johnson and Proctor 2016, pg. 15; bold print added). Johnson and Proctor are here discussing skills in general rather than expertise, but they classify forms of expertise in the same way and rely on such a classification in the chapter of their book dedicated to expertise.

${ }^{2}$ Johnson and Proctor also express hesitancy in the following wording: "Although [radiology and computer programing] rely heavily on knowledge, the diagnosis of medical images has a large perceptual component, whereas computer programming is mainly a cognitive task..." (Johnson and Proctor 2016, pg. 155). And in Yarrow et al's review of expertise in sports one finds: "Consideration of what is required to be good at sport leads to the realization that distinctions between perception, cognition and motor control are fuzzy at best. If maintaining separate domains of perception, cognition and action is useful for heuristic purposes, then evidence suggests that athletes develop practise-dependent task-specific skills in all three domains." (Yarrow et al 2009, pg. 585; bold print added).

${ }^{3}$ One might worry, for example, how (1) there can be a border between perception and cognition, (2) expert perception can be a capacity that manifests itself in superior perceptual experiences rather than just superior perceptual judgments, and (3) expert perception can be a capacity that draws on cognition. I do think the three claims are consistent and discuss this at length elsewhere. The main ideas have been around at least since (Pylyshyn 1999). There he argued that cognitively driven attention and cognitively driven learning of "compiled transducers" are both compatible with perceptual processes not having access to cognitively stored information. I believe that Pylyshyn was correct about this, and that proper understanding of the different roles of attention, learning, and information access support an even stronger form of perceptual modularity than the one he defended. (1), (2), and (3), then, can be consistently maintained, given the further claim that the role of cognition in perceptual expertise is confined to cognitively driven attention and cognitively driven learning of "compiled transducers."

${ }^{4}$ Compare: " . . despite its seeming simplicity, transcription typing involves an intricate and complex interaction of perceptual, cognitive, and motoric processes. Not only does verbal material have to be registered and perceived, but it has to be appropriately partitioned, accurately translated into physical movements, and then those movements executed at rates exceeding several hundred keystrokes per minute." (Salthouse 1986, pg. 303)

${ }^{5}$ Though the notion of search is very general, it is not trivially applicable in psychological explanation. My thesis-that expertise in forming impressions about a domain is a capacity for forming superior impressions about that domain that is grounded in domain related search strategies-constrains psychological accounts of expert impressions to those that take the form of theories about domain related search strategies. Specific empirical predictions must wait on specific hypotheses-e.g. experts in domain $\mathrm{X}$ search widely rather than deeply. Still, one might worry that my thesis about the structure of expertise in forming impressions is implausible outside of domains with a large perceptual componente.g. radiology, chess-or domains with a clearly recognizable problem solving component-e.g. physics, chess-with respect to which search strategies have been extensively theorized. One example is history. (Wineburg 1991) studied how expert historians approached the question of the relative historical accuracy of three different paintings of the Battle of Lexington, with the express motivation of moving research on expertise beyond the familiar paradigms-radiology, chess, physics, etc. Let me register one initial reservation about interpreting this research: Wineburg studied expert/novice differences in how subjects brought source material to bear on answering the question of accuracy; so his study is of a deliberate research activity and may reveal more about a mix of expert actions and expert impressions than expert impressions alone, in which case its significance for assessing my thesis can be difficult to interpret. Nonetheless, in his discussion Wineburg writes: expertise seemed to rest less on bringing the right problem schema to the task and more on constructing a context-specific schema tailored to this specific event (Wineburg 1991, pg. 83). The difference he emphasizes is that between (1) checking accuracy of details in accordance with a schema known to be relevant by recognizing the case's similarity to a familiar case, and (2) checking accuracy of details in accordance with a schema constructed by applying heuristics to the specific case, such as the heuristic of prioritizing details corroborated by multiple sources. This shows something about the contingency of history, but it does not really undermine the framework for thinking about expert impressions that I am endorsing here. The historians searched for those matches and discrepancies in details their expertise enabled them to identify and prioritize. All that said, I am open minded about the existence of expert impressions that do not fit the account I am giving here. In the body of the paper I work with the unrestricted thesis about expert impressions 
in order to keep the discussion simple. But if the thesis ultimately has to be restricted to a subclass of expert impressions, then that would be compatible with my main goals. I am grateful to an anonymous referee for pressing me to think through this issue.

${ }^{6}$ (Ma, Husain, and Bays 2014) review new research on the nature of working memory limitations. Classic discussions of expert memory assumed these limitations take the form of a limited number of discrete slots. New research suggests that these limits take the form of a limited resource that can be distributed among any number of items with continuously diminishing precision. The basic structure of the explanation of expert memory can persist through this change in conception of working memory: for novice mnemonists in a domain individual items compete for the limited working memory resource; for expert mnemonists in a domain larger and larger groups of those items become the units of competition.

${ }^{7}$ I presented some of this material at a workshop on acquaintance and direct grasp organized by Uriah Kriegel at the Jean Nicod Institute in July 2018. Thanks to Uriah and the other participants in the workshop for feedback that induced a sharper if not a direct grasp of the relevant issues. I am very grateful to an anonymous referee for this journal for an incisive set of comments on a previously submitted version of the paper.

\section{Bibliography}

Beck, J. (2017). "Marking the Perception-Cognition Boundary: The Criterion of Stimulus-Dependence." Australasian Journal of Philosophy: 1-16.

Benner, P. (1982). "From novice to expert." AJN The American Journal of Nursing 82(3): 402-407.

Bilalić, M. (2017). The neuroscience of expertise, Cambridge University Press.

Brogaard, B. (2013). Phenomenal Seemings and Sensible Dogmatism. Seemings and Justification. C. Tucker, Oup Usa: 270.

Chase, W. G. and K. A. Ericsson (1982). Skill and working memory. Psychology of learning and motivation, Elsevier. 16: 1-58.

Chase, W. G. and H. A. Simon (1973). "Perception in chess." Cognitive psychology 4(1): 55-81.

Chi, M. T. (2011). Theoretical perspectives, methodological approaches, and trends in the study of expertise. Expertise in mathematics instruction, Springer: 17-39.

Chi, M. T., et al. (1981). "Categorization and representation of physics problems by experts and novices." Cognitive science 5(2): 121-152.

Christensen, W., et al. (2016). "Cognition in skilled action: Meshed control and the varieties of skill experience." Mind \& Language 31(1): 37-66.

Clark, A. (2013). "Whatever next? Predictive brains, situated agents, and the future of cognitive science." Behavioral and brain sciences 36(3): 181-204.

De Groot, A. (1965). Thought and Choice in Chess The Hague, Mouten.

Ericsson, K. A. and N. Charness (1994). "Expert performance: Its structure and acquisition." American Psychologist 49(8): 725.

Ericsson, K. A., et al. (2006). The Cambridge handbook of expertise and expert performance, Cambridge University Press.

Ericsson, K. A., et al. (2018). The Cambridge handbook of expertise and expert performance, Cambridge University Press.

Ericsson, K. A. and W. Kintsch (1995). "Long-term working memory." Psychological review 102(2): 211.

Evans, J. S. B. and K. E. Stanovich (2013). "Dual-process theories of higher cognition: Advancing the debate." Perspectives on psychological science 8(3): 223-241.

Firestone, C. and B. J. Scholl (2016). "Cognition does not affect perception: Evaluating the evidence for" top-down" effects." Behavioral and brain sciences 39.

Fridland, E. (2014). "They've lost control: Reflections on skill." Synthese 191(12): 2729-2750.

Fridland, E. (2017). "Skill and motor control: intelligence all the way down." Philosophical studies 174(6): 1539-1560.

Gauthier, I., et al. (2010). Perceptual expertise: Bridging brain and behavior, OUP USA.

Gentner, D. R. (1988). "Expertise in typewriting." The nature of expertise: 1-21. 
Gobet, F. (2015). Understanding expertise: A multi-disciplinary approach, Macmillan International Higher Education.

Gobet, F. and H. A. Simon (1996). "Templates in chess memory: A mechanism for recalling several boards." Cognitive psychology 31(1): 1-40.

Johnson, A. and R. W. Proctor (2016). Skill Acquisition and Training: Achieving Expertise in Simple and Complex Tasks, Taylor \& Francis.

Kundel, H. L. and C. F. Nodine (1975). "Interpreting chest radiographs without visual search." Radiology 116(3): 527-532.

Lupyan, G. (2015). "Cognitive penetrability of perception in the age of prediction: Predictive systems are penetrable systems." Review of Philosophy and Psychology 6(4): 547-569.

Ma, Wei Ji, Masud Husain, and Paul M. Bays. "Changing concepts of working memory." Nature neuroscience 17, no. 3 (2014): 347.

Mandelbaum, E. (2017). "Seeing and conceptualizing: Modularity and the shallow contents of perception." Philosophy and Phenomenological Research.

McPherson, S. L. and J. R. Thomas (1989). "Relation of knowledge and performance in boys' tennis: Age and expertise." Journal of experimental child psychology 48(2): 190-211.

Montero, B. G. (2016). Thought in action: Expertise and the conscious mind, Oxford University Press.

Nodine, C. F. and H. L. Kundel (1987). "Using eye movements to study visual search and to improve tumor detection." Radiographics 7(6): 1241-1250.

Nokes, T. J., et al. (2010). Problem solving and human expertise. International encyclopedia of education, Elsevier Ltd.

Orlandi, N. (2014). The Innocent Eye: Why Vision is Not a Cognitive Process, Oup Usa.

Palmer, S. E. (1999). Vision science: Photons to phenomenology, MIT press.

Pesenti, M., et al. (1999). "Basic and exceptional calculation abilities in a calculating prodigy: A case study." Mathematical Cognition 5(2): 97-148.

Phillips, B. (2017). "The Shifting Border Between Perception and Cognition." Noûs.

Pylyshyn, Z. (1999). "Is vision continuous with cognition?: The case for cognitive impenetrability of visual perception." Behavioral and brain sciences 22(3): 341-365.

Reingold, E. M. and N. Charness (2005). "Perception in chess: Evidence from eye movements." Cognitive processes in eye guidance:: $325-354$.

Reingold, E. M., et al. (2001). "Visual span in expert chess players: Evidence from eye movements." Psychological Science 12(1): 48-55.

Reingold, E. M. and H. Sheridan (2011). "Eye movements and visual expertise in chess and medicine." Oxford handbook on eye movements: $528-550$.

Salthouse, T. A. (1986). "Perceptual, cognitive, and motoric aspects of transcription typing." Psychological bulletin 99(3): 303.

Sheridan, H. and E. M. Reingold (2017). "The Holistic Processing Account of Visual Expertise in Medical Image Perception: A Review." Frontiers in psychology 8: 1620.

Stanley, J. and J. W. Krakauer (2013). "Motor skill depends on knowledge of facts." Frontiers in human neuroscience 7: 503.

Staszewski, J. J. (1988). "Skilled memory and expert mental calculation." The nature of expertise: 71-128.

Stroop, J. R. (1935). "Studies of interference in serial verbal reactions." Journal of experimental psychology 18(6): 643.

Tanaka, J. W. and M. Taylor (1991). "Object categories and expertise: Is the basic level in the eye of the beholder?" Cognitive psychology 23(3): 457-482.

Toner, J., et al. (2015). "Considering the role of cognitive control in expert performance." Phenomenology and the Cognitive Sciences 14(4): 1127-1144.

Wineburg, S. S. (1991). Historical problem solving: A study of the cognitive processes used in the evaluation of documentary and pictorial evidence. Journal of educational Psychology, 83(1), 73.

Yarrow, K., et al. (2009). "Inside the brain of an elite athlete: the neural processes that support high achievement in sports." Nature Reviews Neuroscience 10(8): 585. 